\title{
MODEL OF DECOMPRESSION MELTING MECHANISM IN CONVECTIVE-UNSTABLE THERMAL LITHOSPHERE (FIRST APPROXIMATION)
}

\section{B.V. Lunev ${ }^{\circledR}$, V.V. Lapkovsky}

Trofimuk Institute of Petroleum Geology and Geophysics, Siberian Branch of the Russian Academy of Sciences, 3 Academician Koptyug Ave, Novosibirsk 630090, Russia

\begin{abstract}
We propose a model of decompression melting, separation, migration and freezing of the melt in the upper mantle during the convective instability process. The model takes into account differences between phase diagrams of the melt and the matrix and the resultant features of the melt's behavior, without calculating reaction rates in a multicomponent medium. It is constructed under an explicit concept of the local thermodynamic equilibrium of the existing phases. Therefore, we further develop the first approximation of the descriptions of convection in the upper mantle and the formation of large epicontinental sedimentary basins, which have been presented in earlier publications.

Our computational experiments show that primary melting of the upper mantle's fertile material occurs intensively in a narrow frontal part of the ascending hot material flow. Then, the depleted and partially melted material rises farther upward from the front of primary melting. Melting of the depleted material continues at lower pressures in a rather wide range of depths (120-77 km). Further, the migrating melt is supplied by two sources, i.e. a deep-seated one, wherein the fertile material melts, and the medium-depth one, wherein melting of the depleted material takes place. Once the temperature and pressure rates of the melt reach the values corresponding to those of its solidus, a narrow freezing front is formed. Its width is almost similar to the primary melting front. As the ascending convective flow develops, the freezing front shifts upward. As a result, a quite thick (around 40-50 km) basalt-saturated layer occurs above the freezing front. An important observation in our modeling experiments is that, despite a considerably large total volume of the melted material, a one-time melt content in the mantle does not exceed tenths of one percent, when we consider averaging to volumes with a linear size of about $1.0 \mathrm{~km}$. The basalt melt extraction depletes iron in the mantle and significantly reduces the mantle density. Considering the calculated basalt-depletion values for the matrix at 0.1-0.2, the density deficit doubles in comparison to the thermal expansion of the material. Logically, both the Rayleigh number and the intensity of convection also double (and this is confirmed by the calculations), which means that convection is enhanced after the melting start.

Testing of the model shows that it gives a reasonable picture that is consistent with the available geological and geophysical data on the structure of the lithosphere underneath the currently developing epicontinental sedimentary basins. Furthermore, within the limits of its detail, this model is consistent with the results of modeling experiments focused on melting and melting dynamics, which are based on calculations of reactions between components of the mantle material.
\end{abstract}

KEY WORDS: decompression melting; numerical modeling; petrological zoning of the upper mantle; mantle convection

FUNDING: The study was performed under the Programme of Basic Scientific Research of the Siberian Branch of RAS (project IX.131.2.2.) and supported by the Russian Foundation for Basic Research (project 18-05-70105).

\section{RESEARCH ARTICLE}

FOR CITATION: Lunev B.V., Lapkovsky V.V., 2021. Model of decompression melting mechanism in convective-unstable thermal lithosphere (first approximation). Geodynamics \& Tectonophysics 12 (3), 485-498. doi:10.5800/GT-2021-12-3-0535 


\title{
МОДЕЛЬ МЕХАНИЗМА ДЕКОМПРЕССИОННОГО ПЛАВЛЕНИЯ В КОНВЕКТИВНО-НЕУСТОЙЧИВОЙ ТЕРМИЧЕСКОЙ ЛИТОСФЕРЕ (ПЕРВОЕ ПРИБЛИЖЕНИЕ)
}

\author{
Б.В. Лунев, В.В. Лапковский
}

Институт нефтегазовой геологии и геофизики им. А.А. Трофимука СО РАН, 630090, Новосибирск, пр-т Академика Коптюга, 3, Россия

АНноТАЦия. Предложена модель декомпрессионного плавления, сепарации, миграции и замерзания расплава в процессе развития конвективной неустойчивости верхней мантии, позволяющая учесть различие фазовых диаграмм расплава и матрицы и вытекающие особенности поведения расплава, без расчета скорости реакций в многокомпонентной среде, в рамках явного представления о локальном термодинамическом равновесии существующих фаз. Таким образом, дополняется развиваемое нами первое приближение описания процесса конвекции в верхней мантии и формирования крупных эпиконтинентальных осадочных бассейнов, опубликованное ранее.

Вычислительными экспериментами показано, что первичное плавление фертильного вещества верхней мантии происходит интенсивно в узком фронте поднимающегося в восходящем потоке горячего вещества. Далее, вверх от фронта первичного плавления, поднимается деплетированное и частично выплавленное вещество. Дальнейшее плавление деплетированного вещества происходит выше, при меньших давлениях в довольно широком диапазоне глубин (120-77 км). Далее мигрирует расплав уже от двух источников - глубинного, где плавится фертильное вещество, и промежуточного, где плавится вещество деплетированное. Достигнув уровня температур и давлений, соответствующих его солидусу, расплав образует фронт замерзания, примерно такой же узкий, как и фронт первичного плавления. По мере развития восходящего конвективного потока фронт замерзания смещается вверх. В результате под ним формируется довольно толстый (около 40-50 км) слой вещества, насыщенного «базальтовым» компонентом. Важным результатом моделирования является то, что, несмотря на значительные общие объемы выплавляющегося вещества, единовременное содержание расплава в мантии, при осреднении на объемы с линейным размером порядка 1 км, не превышает десятых долей процента. Экстракция базальтовой выплавки, в связи с обеднением мантийного вещества железом, существенно снижает его плотность. При рассчитанных значениях обеднения матрицы базальтовым компонентом 0.1-0.2 дефицит плотности удваивается, по сравнению тепловым расширением вещества. Стало быть, удваивается и число Рэлея, и интенсивность конвекции, что мы и видим в расчетах - после начала плавления конвекция усиливается.

Проведенное опробование модели дает разумную картину, согласующуюся как с известной геолого-геофизической информацией о строении литосферы под развивающимися эпиконтинентальными осадочными бассейнами, так и, в рамках своей детальности, - с результатами моделирования плавления и динамики расплава, полученными путем расчета реакций между компонентами мантийного вещества.

КЛЮЧЕВЫЕ СЛОВА: декомпрессионное плавление; численное моделирование; петрологическая зональность верхней мантии; мантийная конвекция

ФИНАНСИРОВАНИЕ: Работа выполнена по программе IX.131.2.2. фундаментальных научных исследований СО РАН; РФФИ, проект № 18-05-70105.

Для ясного понимания проблемы не следует надевать на тощий скелет экспериментальных фактов слишком сложные математические одеяния. Академик Л.А. Арцимович

\section{1. ВВЕДЕНИЕ}

В нашей предыдущей статье [Lunev, Lapkovsky, 2018] предложена модель развития твердотельной конвекции в термической литосфере, в рамках которой адекватно описывается процесс формирования крупных эпиконтинентальных осадочных бассейнов, развивающихся над восходящими конвективными потоками в верхней мантии. Удалось воспроизвести основные черты эволюции и строения как самих осадочных бассейнов (характер и структура осадочного выполнения), так и подстилающей их литосферы. Однако там не рассматривались процессы, связанные с плавлением вещества и дальнейшим поведением расплава. А известно, что в геологической истории наиболее крупных бассейнов магматические процессы проявлены и играют существенную роль. Проведенные нами численные эксперименты с температурой на подошве термической литосферы выше $1500^{\circ} \mathrm{C}$ показали, что в восходящем потоке на глубине около 100-120 км начинается декомпрессионное плавление сухого мантийного вещества. При использовании фазовой диаграммы мантийного вещества, содержащего летучие компоненты, такое плавление начинается на больших глубинах и имеет место при более низких температурах на подошве конвектирующего слоя. Для выявления возможных эффектов, связанных с декомпрессионным плавлением вещества в восходящих потоках мантийной 
конвекции, в модель нужно внести соответствующие дополнения.

Один из подходов к описанию эффектов декомпрессионного плавления вещества в геодинамическом моделировании предполагает, что образующийся расплав перемещается вместе с равновесным плавящимся веществом матрицы. При этом его присутствие определенным образом влияет на реологические и прочие свойства вещества, в целом снижая общую вязкость и ускоряя тем самым продвижение частично расплавленного вещества к поверхности. В качестве примера развития такого подхода можно привести работы [Sharapov et al., 2006; Perepechko, Sharapov, 2014; Polyansky et al., 2012, 2014, 2016, 2017, 2018].

В рамках другого, более близкого нам, подхода полагается, что расплав, по крайней мере частично, отделяется и мигрирует от породившей его матрицы, замерзая вдали от нее. Такие модели развиваются многими авторами на основе описания, предложенного в статье [McKenzie, 1984]. В рамках этого подхода выполнен ряд основополагающих исследований - укажем, например, работы [Schmeling, 2000, 2006, 2010; Sparks, Parmantier, 1993, 1994; и др.]. Плавление и замерзание расплава при этом традиционно рассчитываются, исходя из диаграммы плавкости того или иного лерцолита (степень плавления и связанная с ней скорость плавления - кристаллизации вещества при этом определяется относительно его кривой солидуса). Однако расплав, образующийся в процессе частичного плавления многокомпонентной матрицы, имеет состав, существенно от нее отличный, поскольку плавятся, прежде всего, наиболее легкоплавкие компоненты породы. Эти различия весьма значительны даже для сухого расплава и сильно усугубляются, если расплав содержит летучие компоненты, тем более что при малых объемах выплавки более высокая растворимость летучих в расплаве (на четыре порядка), по сравнению с матрицей, должна приводить к насыщению образующейся жидкости летучими даже при низком содержании их в матрице.

Кроме того, в традиционных моделях миграция расплава рассчитывается, исходя из представлений о его механической фильтрации по связной системе каналов, образуемых жидкими пленками по граням минеральных зерен. Отсюда определяется минимальное содержание расплава 2 \%, при котором его сепаратное движение становится значимым [McKenzie, 1984; Schmeling, 2000, 2010]. Неизвестно, как в действительности происходит миграция расплава (точнее сказать раствора) в мантии. Но с учетом упомянутого насыщения образующейся жидкости летучими и того обстоятельства, что при мантийных температурах и давлениях они находятся в надкритическом состоянии, можно предположить, что миграция такой жидкости может происходить, например, и путем растворения - переотложения вещества матрицы [Zhuse, 1981; Ablesimov et al., 1988; Ablesimov, Zemtsov, 2010; Gorbachev et al., 2017]. В таком случае ограничение на концентрацию расплава - раствора для начала его движения может быть неуместным.

Моделирование взаимодействия жидкости с матрицей при частичном плавлении (как собственно плавления - замерзания, так и движения жидкости относительно матрицы) в таких условиях требует, по-видимому, описания неравновесных реакций в многокомпонентной системе, сопровождающихся фазовыми переходами. В принципе такую возможность дают существующие методы термодинамических расчетов химических реакций в многокомпонентной системе, например R DMC method - Reactive Disequilibrium MultiComponent method, основанный на теории, развитой в работе [Rudge et al., 2011]. Поскольку их применение связано с очень большими объемами вычислений, затрудняющими включение в геодинамические модели, в работах [Keller, Katz, 2016; Keller et al., 2017; Keller, Suckale, 2019; Lichtenberg et al., 2019; Turner et al., 2017; и др.] на базе метода R DMC развит упрощенный подход, в котором вещество мантии представляется состоящим из четырех взаимодействующих генерализованных компонент. Составы расплава и тугоплавкого рестита определяются в результате расчета реакций между этими компонентами на основе теории идеальных растворов. Скорость реакций определяет и скорость плавления замерзания вещества. При этом значения эффективных коэффициентов, определяющих скорости реакций, отыскиваются подбором так, чтобы удовлетворить эмпирическим данным о положении поверхностей солидуса и ликвидуса исследуемой системы. Таким образом, хотя и подразумевается описание неравновесных процессов химических реакций между компонентами, коэффициенты для их расчета определяются по равновесным состояниям, являющимся результатами этих реакций. При этом число подбираемых свободных параметров оказывается довольно большим - по пять параметров для каждого из четырех компонент. Эти замечания не имеют характера критики - разработанный метод очень остроумен, подразумевает ясный физический смысл и дает замечательные результаты, особенно впечатляющие в части формирования каналов движения жидкости и распределения в ней и в матрице летучих компонент.

В настоящей работе предлагается более простой и, возможно, более гибкий метод моделирования декомпрессионного плавления, сепарации, миграции и замерзания расплава, явно исходящий из представлений о равновесии существующих фаз в каждый момент времени, что, по нашему мнению, лучше соответствует развиваемому нами первому приближению описания процесса конвекции в верхней мантии.

\section{2. МЕТОДИКА}

Обозначая $l$ объемную долю присутствующего расплава, $M$ - скорость его производства, $\bar{v}_{s}$ и $\bar{v}_{l}-$ скорость течения матрицы и расплава (так, $\bar{v}_{l}=\bar{v}_{s}+\bar{w}$ что, где $\bar{w}-$ скорость движения расплава относительно матрицы), используя теорему Гаусса - Остроградского и полагая 


$$
\nabla \cdot \bar{v}_{s}+\nabla \cdot \bar{w}=0
$$

имеем:

$$
\int \frac{\partial l}{\partial t} d V=\int M d V-\phi l \bar{v}_{l} \cdot \overline{d s},
$$

$$
\begin{gathered}
\phi l \bar{v}_{l} \cdot \overline{d s}=\int \nabla \cdot\left(l \bar{v}_{l}\right) d V=\int \nabla \cdot\left(l \bar{v}_{s}+l \bar{w}\right) d V= \\
=\int\left(\bar{v}_{s} \cdot \nabla l+\bar{w} \cdot \nabla l\right) d V+\int l\left(\nabla \cdot \bar{v}_{s}+\nabla \cdot \bar{w}\right) d V, \\
\frac{\partial l}{\partial t}=M-\bar{v}_{l} \cdot \nabla l .
\end{gathered}
$$

Положим, что $M=M_{M}+M_{F}$, где $M_{M} \geq 0$ - скорость плавления, а $M_{F} \leq 0$ - скорость замерзания вещества, и запишем уравнение (4) для такого воображаемого расплава I', который выплавляется и мигрирует, но нигде не замерзает:

$$
\frac{\partial l^{*}}{\partial t}=M_{M}-\bar{v}_{l} \cdot \nabla l^{*}
$$

Скорость плавления $M_{м}$ определим, как обычно, чеpeз $l_{s(\chi, P, T)}$ - объемную долю расплава, равновесного для данного состава $\chi$ движущегося элемента матрицы, при данных давлении $P$ и температуре $\Theta$ :

$$
M_{M}=\frac{D_{s} l_{s}}{D t}=\frac{\partial l_{s}}{\partial t}-\bar{v}_{s} \cdot \nabla l_{s} .
$$

Как обсуждалось во Введении, состав выплавившейся жидкости может существенно отличаться от матрицы. Обозначая $l_{l(x, P, T)}$ долю равновесного при данных $P$ и $\Theta$ расплава для состава образующего его вещества, можно определить собственно долю присутствующего расплава:

$$
l=l_{l}{ }^{*}
$$

и скорость замерзания

$$
M_{F}=M-M_{M}=\frac{D_{l} l}{D t}-\frac{D_{s} l_{s}}{D t},
$$

здесь $\frac{D_{s}}{D t}=\frac{\partial}{\partial t}+\bar{v}_{s} \cdot \nabla$ и $\frac{D_{l}}{D t}=\frac{\partial}{\partial t}+\bar{v}_{s} \cdot \nabla$.

Равновесные доли расплава $l_{s}$ и $l_{p}$, если составы вещества матрицы и жидкости в данной точке известны (вычислены или некоторым образом заданы), могут быть определены из соответствующих фазовых диаграмм.

Таким образом, плавление и замерзание вещества определяются в соответствии с составами матрицы и расплава, в рамках представлений о локальном термодинамическом равновесии. (Это представляется существенным, когда речь идет о реализации модели первого приближения, в которой конвектирующее вещество в каждый момент времени считается локально равновесным).

Скорость производства расплава $\left(M=M_{M}+M_{F}\right)$ позволяет рассчитывать степень обеднения - обогащения матрицы базальтовым компонентом (и железом), $B$, существенно изменяющим ее состав в некоторых зонах и влияющим на такой геодинамически важный параметр, как плотность вещества:

$$
\frac{\partial B}{\partial t}=M-\bar{v}_{s} \cdot \nabla B
$$

Величина $B$ может изменяться от -1.0 , что будет соответствовать веществу матрицы с полностью выплавленным базальтовым компонентом, до +1.0, когда вещество матрицы целиком представлено базальтом. Случай $B=0$, очевидно, соответствует фертильной матрице (мантии - в рассматриваемом случае). В дальнейшем будем пользоваться также обозначениями $B_{-}-$ для обозначения степени обеднения матрицы базальтовым компонентом и $B_{+}$- обогащения.

Обычно эту величину называют степенью деплетированности. Мы предпочитаем называть «деплетацией» истощение матрицы концентрирующимися в расплаве компонентами - летучими и другими расплавофильными элементами, происходящее не только путем плавления, но и просто в результате присутствия мигрирующего расплава. В отличие от обеднения базальтовым компонентом, такое истощение практически необратимо, так как при частичной кристаллизации расплавофильные компоненты остаются в расплаве и переходят в состав твердой матрицы только при полном его замерзании, что может иметь место в особых условиях.

Благодаря огромной разности растворимостей, можно считать, что при появлении любого количества расплава все расплавофильные элементы из окрестности переходят в него (во всяком случае, такое упрощение используется). Исходя из этого предположения, для вычисления степени деплетированности D можно записать:

$$
\begin{gathered}
l>0 \rightarrow D=1 \\
l=0 \rightarrow \frac{\partial D}{\partial t}=-\bar{v}_{s} \cdot \nabla D .
\end{gathered}
$$

Это означает, что в отсутствие расплава интенсивность деплетации равна нулю, а если какое-то количество расплава имеется, то он полностью деплетирует окрестность и $D=1$, при любых прочих условиях. Промежуточные значения деплетация $D$ может принимать только благодаря перемешиванию вещества матрицы твердотельным течением $-\bar{v}_{s} \cdot \nabla D$.

Предположение о полной деплетации вещества любым количеством расплава легко может быть скорректировано подстановкой в (10) той или иной зависимости $D_{(\eta)}$. При этом, в принципе, может быть учтена зависимость от состава расплава и характера его локализации.

Концентрацию в матрице какого-либо расплавофильного компонента $g_{s}$ (прежде всего - летучих) можно описать просто:

$$
g_{S}=g_{S}^{0}(1-D)
$$

где $g_{s}^{0}$ - исходное содержание компонента в матрице.

Величины $B, D, g_{s}$ с использованием соответствующих фазовых диаграмм позволяют для каждой точки, с известными в данный момент времени давлением и температурой, определить долю равновесного с матрицей расплава $l_{s}$. 
Чтобы сделать то же в отношении $l_{l}$ - доли расплава, равновесного с составом вещества, образующего жидкость, очевидно, нужно знать его состав, прежде всего - содержание летучих компонент, и изменение их содержания в процессе эволюции расплава. Этот вопрос мы предполагаем рассмотреть в последующих статьях по данной тематике, а в рамках данной работы для проведения численных экспериментов, имеющих целью выяснение общих закономерностей процесса плавления, миграции и замерзании расплава при развитии конвекции в верхней мантии, мы оперировали фазовой диаграммой для некоторого заданного состава расплавленного вещества с фиксированным содержанием летучих, в данном случае - диаграммой водонасыщенного базальта (эклогита).

Скорость движения расплава относительно матрицы $-\bar{w}$, определим, как обычно, при помощи уравнения Дарси (являющегося первым приближением уравнения движения флюида в теории многоскоростного континуума [Blokhin, Dorovsky, 1994]), в зависимости от его плавучести $\Delta \rho_{l} \bar{g}\left(\Delta \rho_{l}-\right.$ дефицит плотности расплава, по сравнению с матрицей, $\bar{g}$-ускорение силы тяжести) и градиента давления $\nabla p$ (точнее сказать - градиента возмущения давления, см. ниже):

$$
\bar{w}=A_{(l)}\left[\nabla p+\Delta \rho_{l} \bar{g}\right] .
$$

Коэффициент $A_{(l)}$ в уравнении (13) обычно определяется как отношение проницаемости матрицы к вязкости расплава на основании представлений о его фильтрации по граням минеральных зерен [McKenzie, 1984]. Однако, как отмечалось во Введении, физика процесса миграции расплава может быть существенно иной, поэтому мы отказались (пока) от конкретизации физического смысла коэффициента $A_{(l)}$ ограничившись градуировкой его зависимости от концентрации расплава (сильно нелинейной - рис. 1), подобранной так, чтобы удовлетворить имеющимся данным прямых и косвенных наблюдений скорости движения базальтового расплава [Kelemen et al., 1997; Batanova, Savelieva, 2009], и убрав ограничение на минимальное содержание расплава (очевидно, что, если потребуется, его легко ввести наложением дополнительных условий на (13)). Заметим, что в диапазоне содержаний расплава, реально участвующем в расчетах, числовые значения нашего коэффициента приблизительно соответствуют таковым у обычно используемого. В дальнейшем эту зависимость можно уточнить на основании тех или иных экспериментальных данных (или теоретических представлений). Очевидно, что включение в модель видоизмененной зависимости затруднений не вызовет.

Постановка задачи расчета твердотельной конвекции в термической литосфере дана в нашей предыдущей статье [Lunev, Lapkovsky, 2018]. С учетом изложенного она принимает следующий вид.

Задача определения твердотельного течения $\bar{v}_{s}$ остается прежней, сформулированной относительно возмущений, с представлением поля плотности, напряжений и давления в виде сумм:

$$
\begin{aligned}
& \rho_{(\bar{x}, t)}=\rho_{i j_{(\bar{x}, t)}}^{0}+\sigma_{(\bar{x}, t)}, \\
& T_{i j_{(\bar{x}, t)}}=T_{i j_{\left(x_{3}, t\right)}^{0}}^{0}+\tau_{i j_{(\bar{x}, t)}} P_{(x, t)}=P_{\left(x_{3}, t\right)}^{0}+P_{(\bar{x}, t)},
\end{aligned}
$$

где $\rho_{\left(x_{3}, t\right)}^{0}, T_{i j_{(x, t)}}^{0}$ и $P_{\left(x_{3}, t\right)}^{0}$-характеристики гидростатического состояния, а $\sigma_{(\bar{x}, t)}, \tau_{i j_{\bar{x}, t]}}$ и $p_{(\bar{x}, t)}-$ возмущения, связанные с течением.

Гидростатическая часть поля напряжений определяется как

$$
T_{i j}^{0}=-\delta_{i j} P^{0}=-\delta_{i j} g \int_{0}^{x_{3}} \rho^{0} d x_{3} .
$$

А для определения ползущего твердотельного течения имеем задачу:

$$
\begin{gathered}
\nabla \cdot \bar{v}_{s}=0, \\
\mu \nabla^{2} \bar{v}_{s}-\nabla p=-\sigma \bar{g}, \\
\left(\nu_{s_{3}}=\tau_{31}=\tau_{32}=0\right)_{x_{3}=0},
\end{gathered}
$$

с дополнительным уравнением, определяющим рельеф свободной поверхности:

$$
\tau_{33_{\left(x_{1}, x_{2}, x_{3}=0\right)}}=-g \int_{0}^{h_{\left(x_{1}, x_{2}\right)}} \rho^{t} d x_{3} .
$$

Уравнение теплопроводности, определяющее изменение температуры $\Theta$, модифицируется с учетом образования, замерзания и движения расплава:

$$
\begin{gathered}
\frac{\partial \Theta}{\partial t}+(1-l) \bar{v}_{s} \cdot \nabla \Theta+l \bar{v}_{l} \cdot \nabla \Theta=\nabla k \nabla \Theta- \\
-\frac{\alpha g}{c_{p}} \Theta \cdot\left[(1-l) v_{s_{z}}+l v_{l_{z}}\right]+\frac{1}{c_{p}} q_{r} \frac{L}{c_{p}} M+\frac{1}{c_{p}} \sum q_{n},
\end{gathered}
$$

дополняясь прежними [Lunev, Lapkovsky, 2018] условиями на границах термической литосферы:

$$
\Theta_{\left(x_{3}=0\right)}=0{ }^{\circ} \mathrm{C}, \Theta_{\left(x_{3}=-300\right)}=1300-1600{ }^{\circ} \mathrm{C}
$$

и начальным распределением температуры

$$
\Theta=\Theta_{(\bar{x})}^{0} .
$$

Здесь $k$ - температуропроводность, $c_{p}$ - удельная теплоемкость и $q_{r}$ - мощность радиоактивного выделения тепла на единицу массы. Член $\frac{1}{c_{p}} \sum q_{n}$ предполагает описание других возможных источников тепла, таких, например, как эффекты твердотельных фазовых переходов и химических реакций (забегая вперед, сразу скажем, что в настоящей работе эти тепловые эффекты не учитывались).

Замыкающее задачу уравнение состояния, определяющее плотность $\rho_{(\bar{x})}$, гидростатическую плотность $\rho_{\left(x_{3}\right)}^{0}$, возмущение плотности $\sigma_{(\bar{x})}$ и дефицит плотности расплава, по сравнению с матрицей $\Delta \rho_{l}$, в общем случае можно записать как

$$
\rho_{(\bar{x})}=\sum_{i}^{n} c_{i} \rho_{i}
$$

где $c_{i}$ и $p_{i}$ - концентрация и плотность каждой из $n$ компонент состава вещества (в соответствии с принятым его представлением), так что $\sum_{i}^{n} c_{i}=1$, а $\rho_{i}=\rho_{i\left(P^{0}, \Theta\right)}$. Если под компонентами понимаются те или иные минеральные ассоциации (генерализованные фазы), то можно записать $\rho_{i\left(P^{0}, \Theta\right)}=\rho_{i}^{0}\left(1+\beta \Delta P^{0}-\alpha_{i} \Delta \Theta\right)$, где 
$\rho_{i}^{0}$ - плотность $i$-й фазы при атмосферных условиях, а $\Delta P^{0}$ и $\Delta \Theta-$ отклонения гидростатического давления и температуры в данной точке $\{\bar{x}\}$ от их значений на поверхности.

В данном случае мы, как и в предыдущей работе, считали вещество коры однородным, а вещество мантии - представленным лерцолитом, с выделением трех обобщенных фаз - гранатового, шпинелевого и плагиоклазового лерцолита с возможным присутствием (в случае $B>0$ ) базальтовой компоненты. Определение фазы вещества мантии производилось соотнесением вычисленных в данной точке значений $\left(P^{0}, \Theta\right)$ с обобщенной фазовой диаграммой лерцолита по [Saxena, Eriksson, 1985; Sobolev, Babeyko, 1994; Litasov, 2011], c учетом существования зон перехода между фазами (диаграммы сухого и влажного лерцолита и базальта-эклогита используются в пропорции, определяемой для каждой расчетной точки на основании вычисленных значений деплетированности $D$ и обеднения/обогащения матрицы базальтовым компонентом $B$ ). Из [Sobolev, Babeyko, 1994] взяты и плотности фаз в поверхностных условиях. Значения параметров вещества задавались для всех фаз одинаковыми и постоянными: коэффициенты теплового расширения $\alpha=10^{-5} \mathrm{~K}^{-1}$, изотермического сжатия $\beta=9 \cdot 10^{-12}$ Па $^{-1}$, температуропроводности $k=$ $=10^{-6} \mathrm{M}^{2} / \mathrm{c}$, удельной теплоемкости $c_{p}=1100 \frac{\text { Дж }}{\mathrm{\kappa} \cdot \mathrm{K}}$ и вязкости матрицы (в уравнении (16)) $\mu=2.5 \cdot 10^{21}$ Па·с, так же как и мощность радиоактивного выделения тепла на единицу массы мантийного вещества $q_{r}=3 \cdot 10^{-12} \frac{\text { Дж }}{\kappa^{*} \mathrm{c}}$.

Дифференциация вещества коры - различия и изменения химического и фазового состава - в расчет пока не принималась, ему была приписана единая плотность в поверхностных условиях 2700 кг/м³.

Чтобы учесть влияние на плотность изменения состава мантии за счет обеднения/обогащения матрицы базальтовым компонентом и присутствия расплава,

(a)

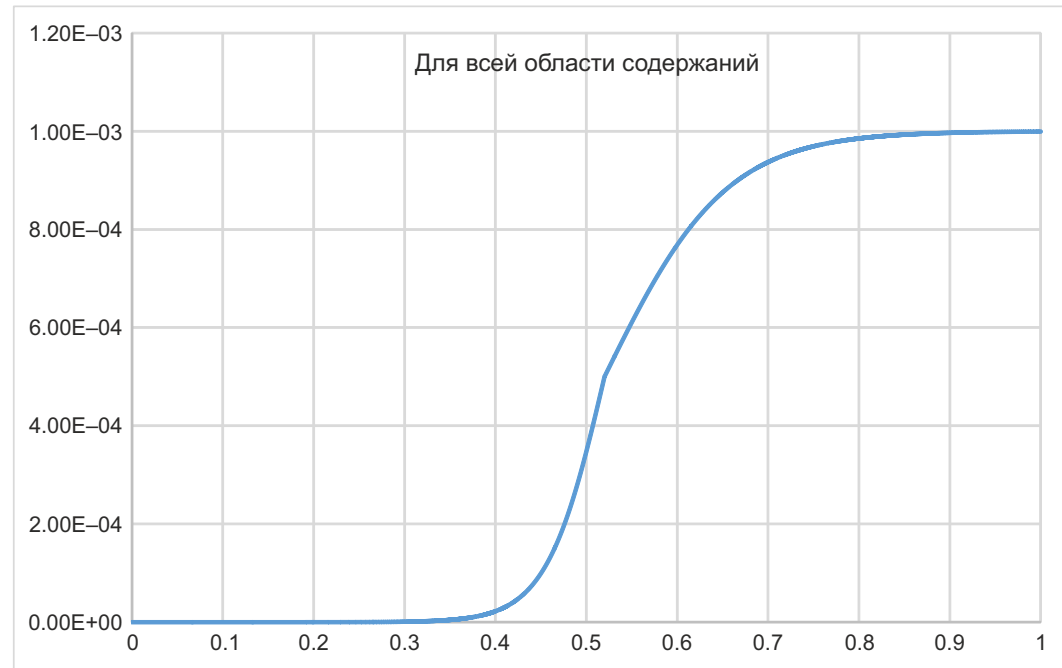

(б)

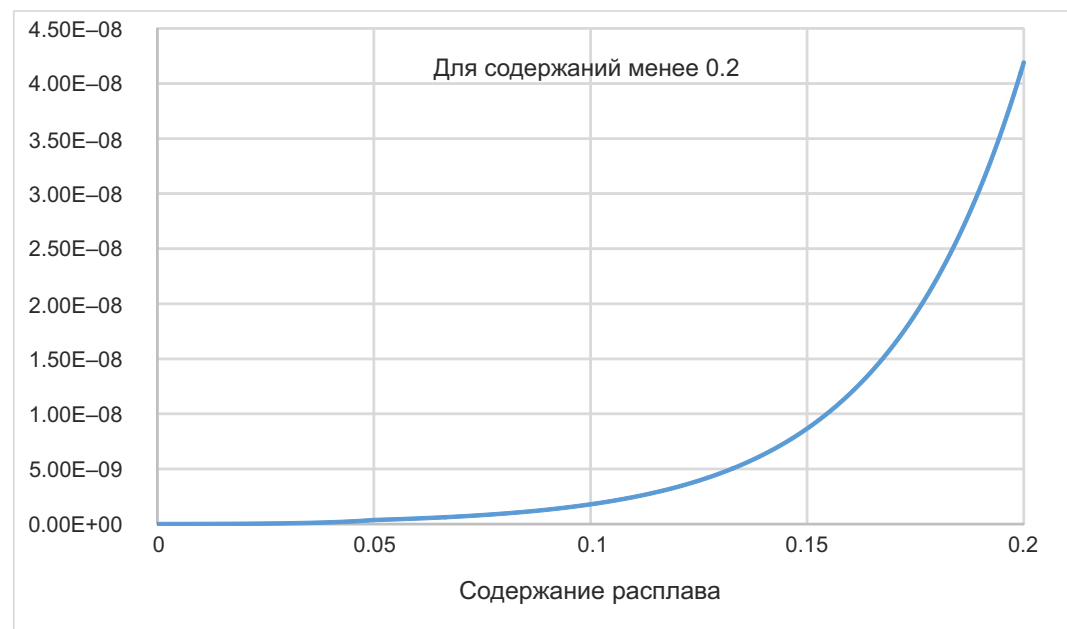

Рис. 1. Зависимость значения коэффициента $A_{(l)} \frac{\mathrm{M}^{3} \mathrm{c}}{\mathrm{\kappa}}$ в уравнении (13) от объемного содержания расплава $l$.

$(a)$ - для всех значений содержания расплава; (б) - детализированный участок графика $(a)$, для области содержаний расплава, реально участвующих в расчетах.

Fig. 1. Dependence of coefficient $A_{(l)} \frac{\mathrm{m}^{3} \mathrm{~s}}{\mathrm{~kg}}$ in equation (13) on volumetric content of melt (I).

Curve $(a)$ is constructed for the entire range of melt content values. (б) - details of curve $(a)$ for the range of melt content values used in calculations. 
в данной работе использовались следующие соотношения:

$$
\begin{gathered}
\rho_{(\bar{x})}=\rho_{s}\left(1-B_{+} l\right)+\rho_{B} B_{+}+\rho_{l} l, \\
\rho_{S(\bar{x})}=\rho_{s_{i}}^{0}\left(1+\beta \Delta P_{\left(x_{3}\right)}^{0}-\alpha \Delta \Theta_{(\bar{x})}-\gamma B_{-(\bar{x})},\right.
\end{gathered}
$$

где $\rho_{s}$ - плотность вещества мантии (без расплава $l$ и без наплавленного вещества базальтового состава $\left.B_{+}\right), \rho_{s_{i}}^{0}-$ плотность при атмосферных условиях $i$-й фазы - гранатового, шпинелевого и плагиоклазового лерцолита (определяемая соотнесением вычисленных в данной точке условий $\left(P^{0}, \Theta\right)$ с фазовой диаграммой лерцолита, см. выше), а $\gamma$ - коэффициент уменьшения плотности вещества в результате обеднения его железом при плавлении. Вообще коэффициент $\gamma$ для каждой фазы свой, но поскольку в рассматриваемой задаче плавится практически всегда гранатовый лерцолит, то мы здесь положили его соответствующей константой $\gamma=0.06$ [Sparks, Parmantier, 1993].

Плотность наплавленного вещества определяли, как для базальта, -

$$
\rho_{B_{+(\bar{x})}}=\rho_{B}^{0}\left(1+\beta_{B} \Delta P_{\left(x_{3}\right)}^{0}-\alpha_{B} \Delta \Theta_{(\bar{x})}\right) .
$$

Плотность расплава также определялась по свойствам базальтового расплава:

$$
\rho_{l_{(\bar{x})}}=\rho_{l}^{0}\left(1+\beta_{l} \Delta P_{\left(x_{3}\right)}^{0}-\alpha_{l} \Delta \Theta_{(\bar{x})}\right) .
$$

Вместе с приведенными выше выражениями (5)(13), определяющими производство, количество и скорость миграции расплава, степень обеднения/обогащения матрицы базальтовым компонентом и степень деплетированности вещества, уравнения (14)-(25) определяют модель конвекции термической литосферы с учетом плавления ее вещества.

\section{3. РЕЗУЛЬТАТЫ МОДЕЛИРОВАНИЯ}

Как и в предыдущей работе [Lunev, Lapkovsky, 2018], моделировалась конвекция континентальной термической литосферы в области $3000 \times 300$ км по сетке $3.0 \times 1.5 \mathrm{\kappa м}$

На рис. 2 представлены результаты расчетов для сухой мантии с температурой на нижней границе $1550{ }^{\circ} \mathrm{C}$, перекрытой сверху исходно 35-километровой континентальной корой.

Декомпрессионное плавление начинается на глубине около 100 км, когда температура там составляет $1450^{\circ} \mathrm{C}$ (рис. 2 , а). Это соответствует состоянию конвекции, при котором восходящий поток мантийного вещества практически достиг поверхности Мохоровичича (изогнутая красная линия на рисунках) и начал формироваться погранслой. Далее, вместе с расширением восходящего потока, область присутствия расплава расширяется, увеличивается и интервал ее глубин - до 120-55 км (рис. 2, б), соответственно продолжающемуся подъему температуры в верхних горизонтах мантии. Затем, при распаде начального восходящего потока на два, разделяется и область присутствия расплава, причем размер каждой из двух новых областей уменьшается как по латерали (соответственно ширине новых восходящих потоков), так и по глубине (в связи с их меньшей интенсивностью), занимая интервал 110-70 км (рис. 2, в). После выхода конвекции в квазистационарный режим доля расплава в сформировавшихся восходящих потоках сухого мантийного вещества изменяется незначительно (рис. 2, г). (Высокую температуру в восходящих потоках по краям расчетной области, обусловливающую присутствие там большей доли расплава, мы склонны относить на счет краевых эффектов).

Более подробно эффекты плавления показаны для стадии 190 миллионов лет на рис. 3.

Видно (рис. 3, б), что плавление происходит в двух зонах: в нижней, с интервалом глубин (на оси восходящего потока) 120-77 км, с максимумом 0.003 млн лет ${ }^{-1}$ на глубине 103 км, и более интенсивное, с максимальными значениями 0.015 млн лет ${ }^{-1}$ - в верхней, на глубинах 65-60 км. По пути миграции расплава, произведенного в нижней зоне первичного декомпрессионного плавления, во всей области его (расплава) присутствия, расчет показывает наличие спорадически распределенных узлов сетки с ненулевой мощностью замерзания, но очень незначительной - порядка $10^{-17}-10^{-18}$ млн лет $^{-1}$. В основном же, как видно из рис. 3 , в, замерзание с мощностью порядка $10^{-2}$ млн лет ${ }^{-1}$ происходит в очень узкой зоне, практически совпадающей с верхней зоной плавления, в которой переплавляется ранее замерзшее вещество базальтового состава. В промежутке между нижней и верхней зонами плавления расплав присутствует в процессе миграции, причем содержание единовременно присутствующего расплава везде очень мало - меньше сотой доли процента. В результате плавления и замерзания на глубинах 120-66 км формируется широкая линза выплавленного, деплетированного вещества, а выше нее, на глубинах 65-40 км, - линза наплавленного вещества, насыщенного базальтовым компонентом (рис. 3 , д), которое вновь подвергается частичной переплавке.

Более выразительную картину дает расчет для влажной мантии, вещество которой содержит $0.01 \%$ воды, с использованием фазовой диаграммы из [Litasov, 2011] (рис. 4). Для расплава пока использована диаграмма водонасыщенного базальта (эклогита).

Как и следовало ожидать, плавление в этом случае начинается на большей глубине - 150 км и, соответственно, раньше - 154 млн лет от начала эволюции. Далее, с подъемом горячего вещества, глубина начального декомпрессионного плавления фертильной мантии еще увеличивается, а глубина замерзания расплава уменьшается.

Общий характер плавления, миграции, замерзания расплава и сопутствующих эффектов рассмотрим на примере состояния системы на момент 174 млн лет от начала эволюции (рис. 4).

Первичное плавление фертильной мантии локализуется в очень узкой 5-километровой зоне, практически - фронте, на глубине около 175 км в развитом 
(a)

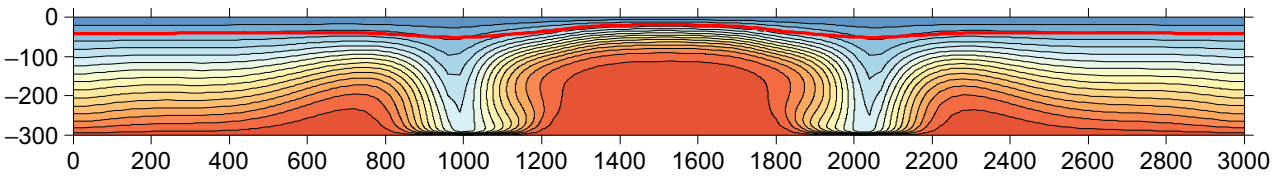

(б)

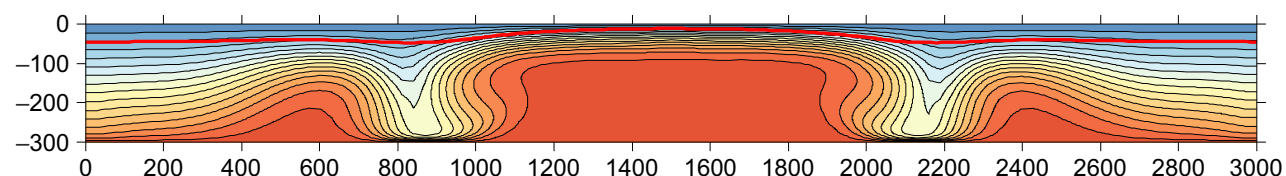

(в)

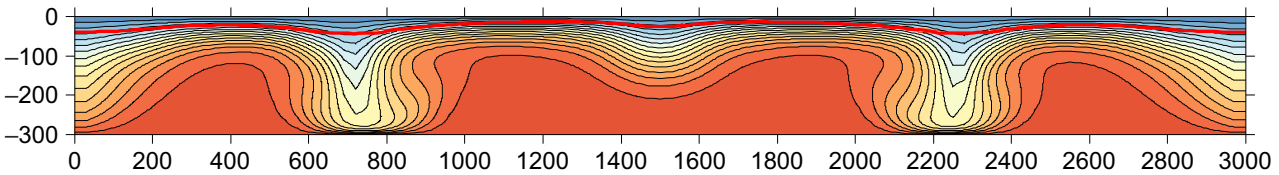

(2)

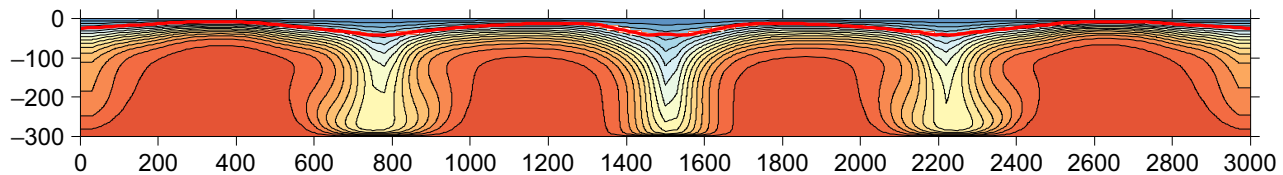

Температура

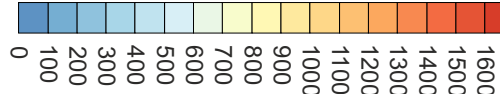

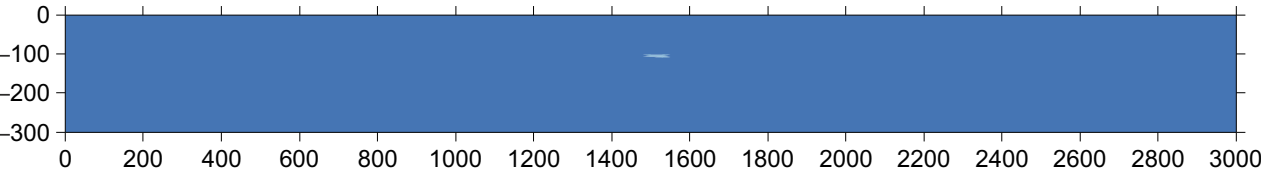
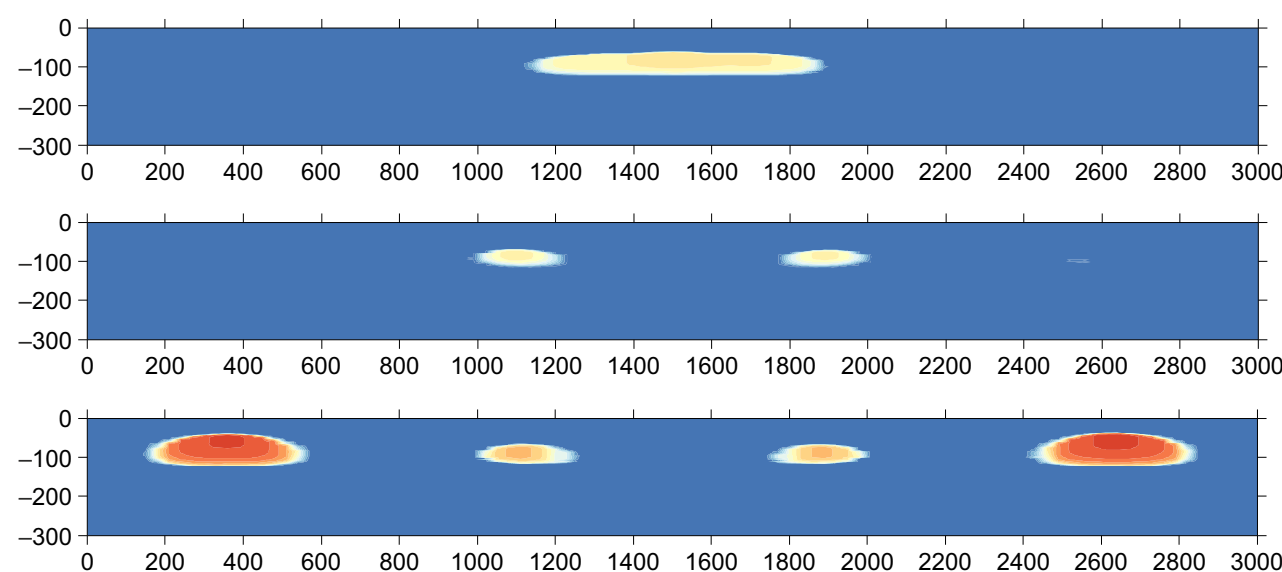

Десятичный логарифмм доли расплава

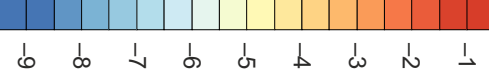

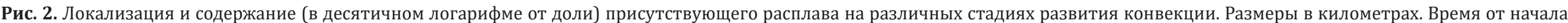
процесса: (a) - 170 млн лет; (б) - 190 млн лет; ( $($ ) - 234 млн лет; (2) - 261 млн лет. Красная линия на рисунках - положение границы Мохоровичича.

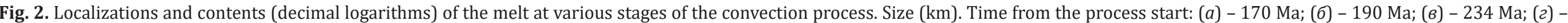
261 Ma. Red line - Moho. 
(d)
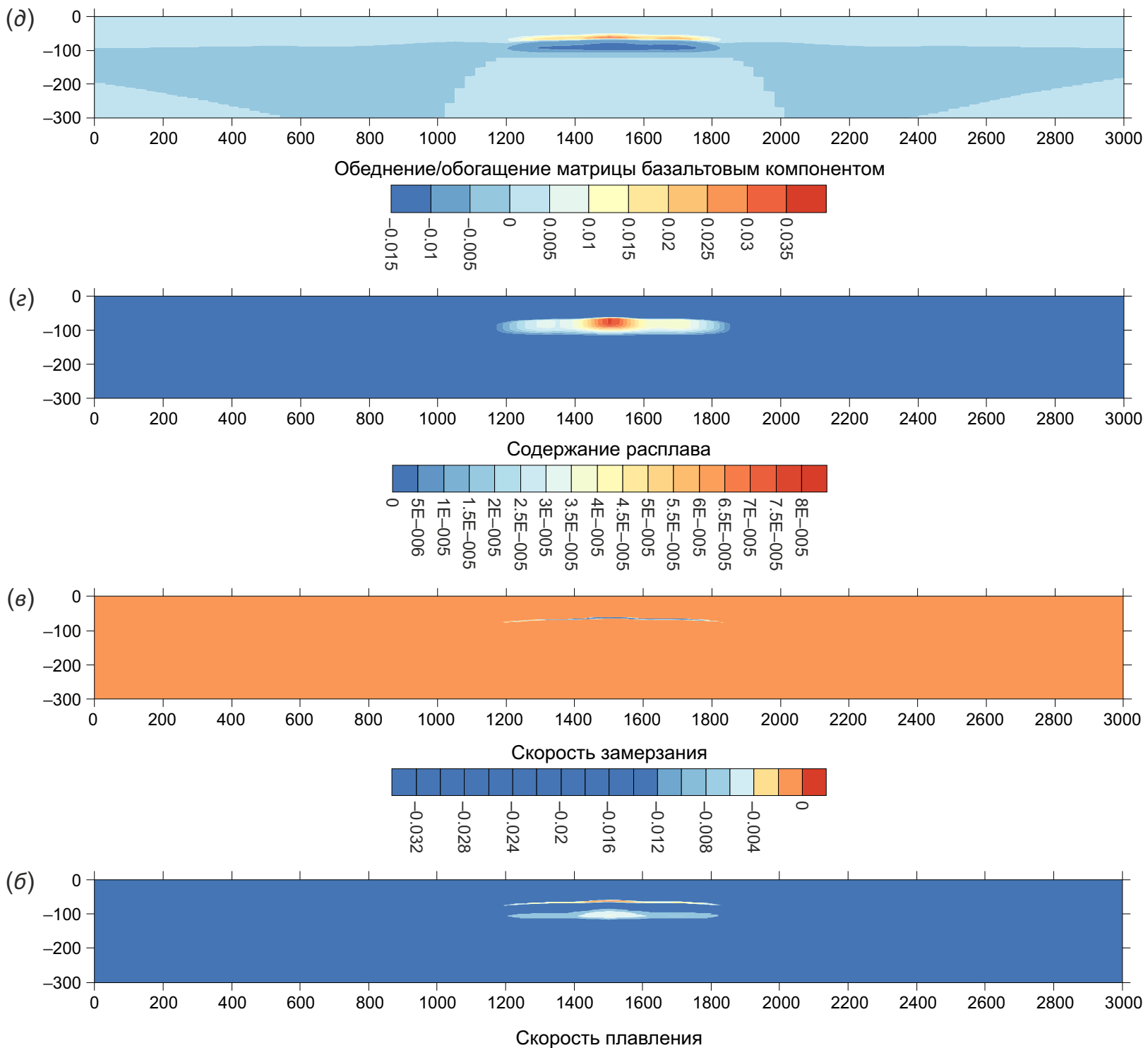

Скорость плавления
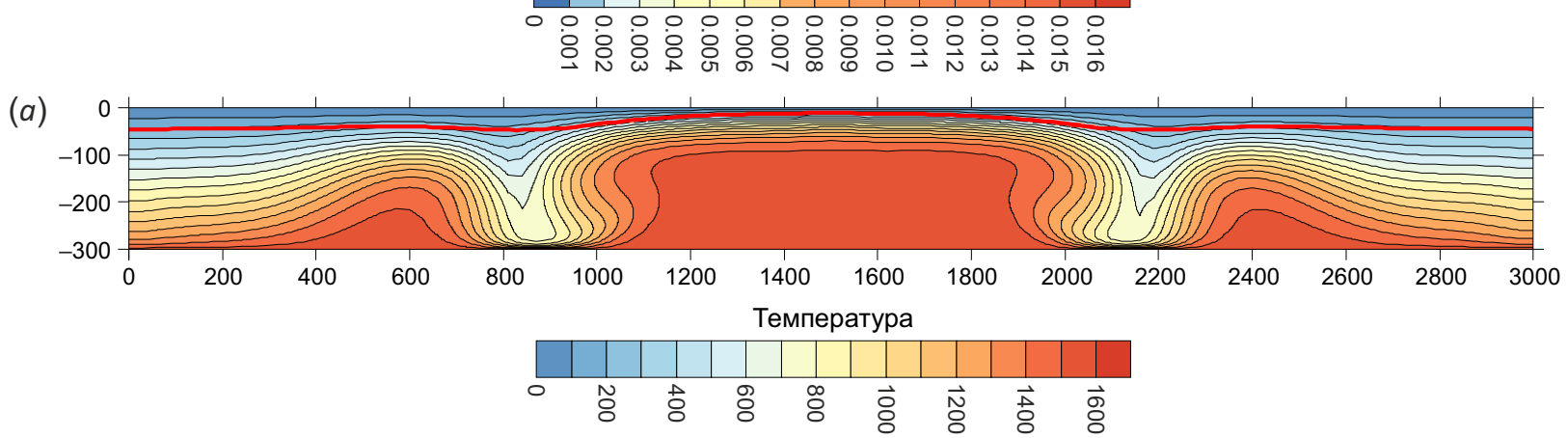

Рис. 3. Эффекты, обусловленные декомпрессионным плавлением сухой мантии, сепарацией, миграцией и замерзанием расплава на стадии 190 млн лет.

(a) - температура; (б) - скорость плавления; (в) - скорость замерзания $\left((б, 8)\right.$ - в объемных долях за миллион лет - млн лет $\left.{ }^{-1}\right)$; (2) - доля расплава; (d) - обеднение/обогащение матрицы базальтовым компонентом ((2, d) - в объемных долях). Красная линия на рисунке $(a)$ - положение границы Мохоровичича.

Fig. 3. Effects due to decompression melting of the 'dry' mantle, separation, migration and freezing of the melt at the stage of $190 \mathrm{Ma}$. $(a)$ - temperature; (б) and ( 8$)$ - melting and freezing rates, respectively (volume fraction per $\left.\mathrm{Ma}^{-1}\right)$; ( 2$)$ - melt fraction; $(\partial)$ - depletion/ enrichment with basalt component (volume fraction). Red line in Fig. (a) - Moho. 
(d)
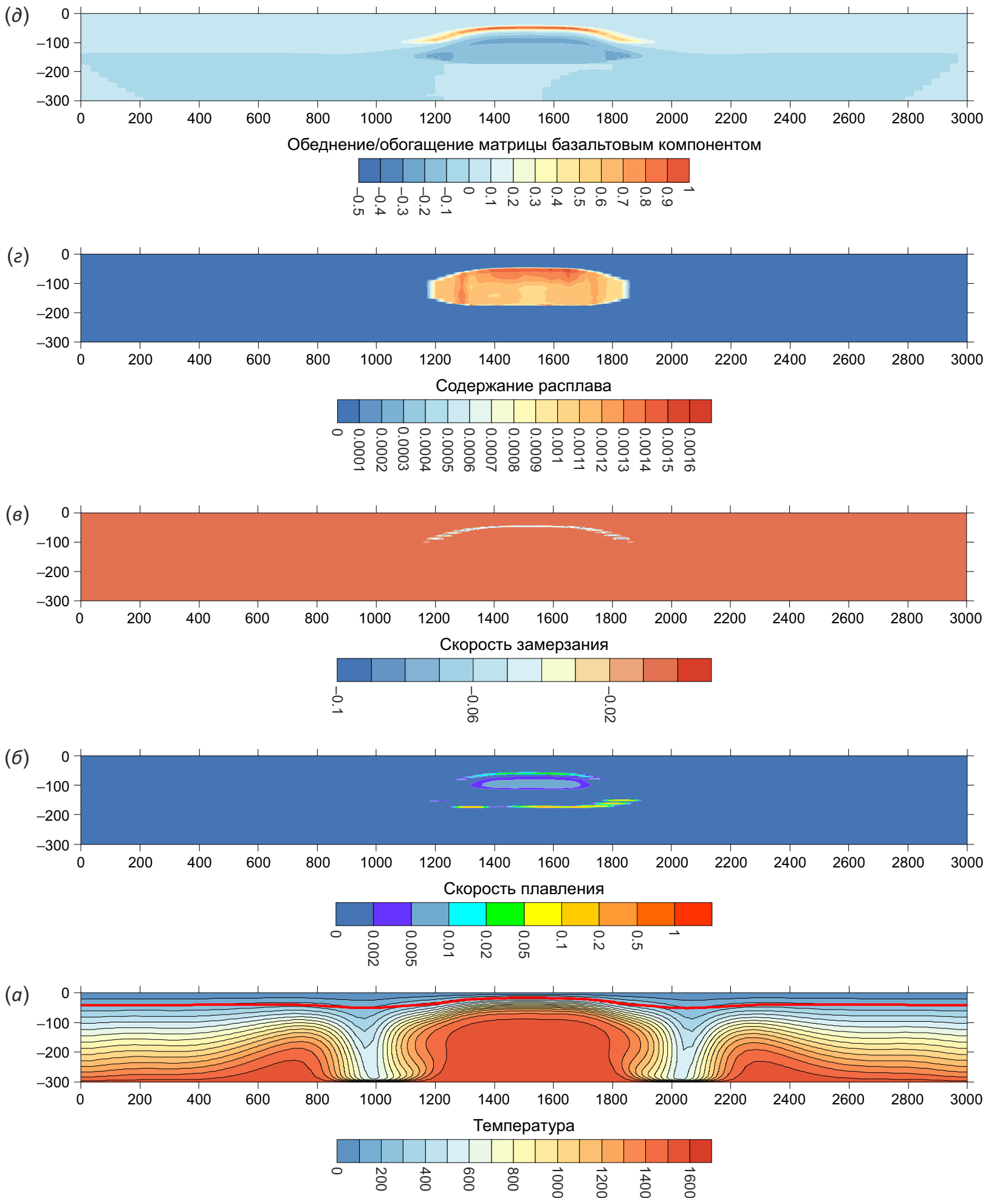

Рис. 4. Эффекты, обусловленные декомпрессионным плавлением влажной мантии, сепарацией, миграцией и замерзанием расплава на стадии 174 млн лет.

(a) - температура; (б) - скорость плавления; (в) - скорость замерзания $\left((б, 8)\right.$ - в объемных долях за миллион лет - млн лет $\left.{ }^{-1}\right)$; (2) - доля расплава; (d) - обеднение/обогащение матрицы базальтовым компонентом ((2, d) - в объемных долях). Красная линия на рисунке $(a)$ - положение границы Мохоровичича.

Fig. 4. Effects due to decompression melting of the 'wet' mantle, separation, migration and freezing of the melt at the stage of $190 \mathrm{Ma}$ $(a)$ - temperature; (б) and ( 8 ) - melting and freezing rates, respectively (volume fraction per $\left.\mathrm{Ma}^{-1}\right)$; ( 2 ) - melt fraction; ( $\partial$ ) - depletion/ enrichment with basalt component (volume fraction). Red line in Fig. (a) - Moho. 
восходящем потоке (рис. 4, б). Скорость плавления в этой зоне гораздо выше, чем в случае сухой мантии, и достигает величин порядка 0.1 млн лет ${ }^{-1}$ при фоновых значениях порядка 0.01 млн лет ${ }^{-1}$ (т.е. примерно в 100 раз мощнее, чем в сухой мантии, хоть и в узкой зоне).

Вверх от фронта первичного плавления поднимается уже деплетированное вещество, которое при данных РТ-условиях не плавится. Зона его плавления расположена выше - от 120 до 65 км, с максимумом на глубинах 100-95 км, где мощность плавления достигает величин около 0.008 млн лет ${ }^{-1}$ (рис. 4, б). Как и следовало ожидать, глубина и интенсивность плавления деплетированного вещества соответствуют тем, что были получены в модели сухой мантии (мощность плавления в этом случае в 2.5 раза выше).

Еще выше, на глубинах 65-55 км на оси восходящего потока, располагается зона вторичного плавления вещества (рис. 4, б), обогащенного ранее замерзшим здесь базальтовым компонентом (рис. 4, д). Скорость плавления здесь имеет тот же порядок, что и для «сухой» мантии, увеличиваясь, по сравнению с ней, примерно вдвое - до 0.025 млн лет ${ }^{-1}$ (рис. 4, б).

Фронт замерзания (очень узкий) располагается непосредственно над зоной вторичного плавления, в интервале глубин на оси восходящего потока 48-43 км (рис. 4, в). Максимальные значения скорости замерзания достигают величины 0.6 млн лет ${ }^{-1}$, что в 20 раз больше, чем для сухой мантии. Так же, как и в случае сухой мантии, расчет показывает замерзание расплава с крайне малой интенсивностью в узлах сетки, спорадически распределенных по всей области его присутствия (см. ниже).

Между фронтом первичного плавления фертильного вещества и фронтом замерзания располагается область присутствия расплава (рис. 4, г). В нижней части области, от 170 до 120 км, присутствует, исключительно в состоянии миграции, первичный расплав фертильного вещества; в зоне от 120 до 65 км к нему добавляется расплав, производящийся из деплетированного вещества, и, наконец, в области от 65 км до фронта замерзания добавляется расплав, образующийся в результате вторичного плавления. Видна неравномерность содержания расплава, отражающая структуру его производства, миграции и скопление в верхней части области. Несмотря на значительное производство расплава, особенно во фронте первичного плавления фертильного вещества, его единовременное содержание, хоть и превосходит таковое для сухой мантии примерно на порядок, все же остается мизерным - меньше $0.1 \%$ (по объему).

Картина обеднения/обогащения матрицы базальтовым компонентом (рис. 4, д) соответствует локализации процессов плавления и замерзания расплава, с учетом изменений в процессе развития конвекции и перемешивания вещества общим конвективным течением. В восходящем потоке, выше 175 км (глубина начала декомпрессионного плавления), формируется обширная линза вещества с различной степенью обеднения базальтовым компонентом: -0.1 от фронта плавления фертильного вещества до начала зоны плавления деплетированного вещества (120 км), где он увеличивается до -0.2. Над выплавленным веществом - от 65 км и вплоть до границы «М»-располагается изогнутый слой с повышенным содержанием замерзшего базальтового компонента, в середине которого степень обогащения базальтовым компонентом достигает 1.0, т.е. полного замещения веществом «базальтового» состава. Наличие этого слоя обеспечивает существование вышеупомянутой локализованной зоны вторичного плавления.

В дальнейшем, с подъемом температуры в восходящем потоке, уменьшается глубина положения фронта замерзания до 20 км, соответственно увеличивается интервал глубин присутствия расплава - 175-20 км. Сдвигаются вверх и другие границы, кроме, естественно, фронта первичного плавления и подошвы зоны плавления деплетированного вещества. Общая картина плавления и поведения расплава принципиально не меняется, за исключением того, что максимум плавления деплетированного вещества разделяется на два, тяготеющих к краям расширяющегося восходящего потока. Соответствующим образом расширяются зоны плавления и замерзания, область присутствия расплава и линзы обедненного и обогащенного базальтовым компонентом вещества.

В целом, при одинаковой температуре нижней границы конвектирующего слоя интенсивность конвекции и всех сопутствующих процессов во влажной мантии оказывается выше, чем в сухой. Конвекция усиливается после начала плавления и, как будет обсуждаться ниже, именно из-за него.

\section{4. ОБСУЖДЕНИЕ РЕЗУЛЬТАТОВ}

Суммируем основные результаты моделирования декомпрессионного плавления и связанных с ним эффектов при конвекции термической литосферы.

Первичное плавление фертильного вещества верхней мантии происходит очень интенсивно, но в узком фронте, расположенном там, где поднимающееся в восходящем потоке горячее вещество достигает соответствующего уровня давления. (В данном случае при температуре подошвы конвектирующего слоя $1550{ }^{\circ} \mathrm{C}$ фронт плавления фертильного вещества располагается на глубине 175 км). Далее, вверх от фронта первичного плавления, поднимается максимально деплетированное ( $D$ до 1$)$ и частично обедненное базальтовым компонентом (B_до -0.1) вещество. Заметим, что именно сопутствующая деплетация фертильного вещества обусловливает узость зоны первичного плавления. Дальнейшее плавление деплетированного вещества происходит выше, при меньших давлениях в довольно широком диапазоне глубин (в данном случае 120-77 км), где степень выплавленности достигает уже -0.2. Расположение зоны плавления деплетированного вещества соответствует локализации зоны первичного плавления для сухой мантии. Тот же порядок, что и для сухой 
мантии, имеет и мощность плавления в этой зоне. Собственно, так и должно быть (ведь здесь плавится уже деплетированное, т.е. «сухое», вещество), что говорит о верности модели и расчетов.

Далее мигрирует расплав уже от двух источников глубинного, где плавится фертильное вещество, и промежуточного, где плавится вещество деплетированное. Достигая уровня температур и давлений, соответствующих его солидусу, расплав образует фронт замерзания, примерно такой же узкий, как и фронт первичного плавления. Поскольку по мере развития восходящего конвективного потока горячее вещество поднимается все ближе к поверхности (пока не сформируется погранслой), фронт замерзания смещается вверх. В результате под ним формируется довольно толстый (около 40-50 км) слой вещества, насыщенного «базальтовым» компонентом. По той же причине (подъем температуры по мере развития восходящего потока конвекции) под фронтом замерзания формируется зона вторичного плавления ранее замерзшего вещества базальтового состава (со скоростью того же порядка, что и в случае сухой мантии, что также ожидаемо). Переплавкой в этой зоне материала, поступившего из трех источников плавления (глубинного фертильного вещества, промежуточного деплетированного и ранее переплавленного), может объясняться как стабильность состава основной массы базальтовых излияний на континентах, так и их известная специфика - обогащение элементами, свидетельствующими о глубинном источнике расплавов [Sobolev et al., 2009]. При этом, судя по структуре миграции расплава (рис. 4, г), можно предположить отдельное замерзание глубинного расплава в краевых частях восходящего потока (чтобы исследовать этот вопрос, требуется ввести в расчеты определение состава расплава, в зависимости от обстоятельств его производства и миграции, как это сделано, например, в работе [Keller at al., 2017], где такой результат и получен).

Важным результатом моделирования является то, что, несмотря на значительные общие объемы выплавляющегося вещества, единовременное содержание расплава в мантии, при осреднении на объемы с линейным размером порядка 1 км, не превышает десятых долей процента и характеризуется неравномерностью, обусловленной как различной интенсивностью плавления и замерзания (в зависимости от состава вещества, температуры и давления), так и нелинейностью процесса его миграции.

Полученные результаты - малое единовременное содержание расплава в системе, наличие различных зон плавления вещества и удаленного от этих зон фронта замерзания расплава - являются прямым следствием учета различий фазовых диаграмм расплава и матрицы, что достигается в модели использованием формул (5)-(8), в сочетании с (13). (Заметим, что подстановка в (13) требования некоторого минимального содержания расплава, необходимого для его движения, относительно матрицы, приводит лишь к тому, что концентрация расплава в зоне его присутствия ограничивается этой величиной).

Примечательно то, что эти наши результаты, полученные в рамках довольно простой феноменологической модели первого приближения, согласуются с результатами моделирования, основанного на расчете реакций между компонентами мантийного вещества [Keller et al., 2017]. Правда, использованная нами довольно редкая расчетная сетка $(3.0 \times 1.5$ км) не позволила получить структуру миграции расплава в виде каналов, как в детальных расчетах [Keller, Katz, 2016; Keller et al., 2017], где используется сетка $0.5 \times 0.5$ км.

Плавление, сепарация, миграция и замерзание расплава изменяют состав мантийного вещества в восходящем конвективном потоке: над фронтом первичного плавления образуется мощный слой деплетированного и частично выплавленного вещества со степенью обеднения базальтовым компонентом, нарастающей вверх по разрезу, над которым располагается слой вещества, насыщенного базальтовым компонентом в результате замерзания мигрировавшего сюда расплава. Обеднение базальтовым компонентом, в связи с обеднением вещества железом, существенно снижает его плотность. В рассматриваемом случае при значениях $B$ $-0.1 \ldots-0.2$ и коэффициенте уменьшения плотности при плавлении гранатового лерцолита $\gamma=0.06$ дефицит плотности удваивается по сравнению с тепловым расширением вещества. Стало быть, удваиваются и число Рэлея, и интенсивность конвекции, что мы и видим в расчетах - после начала плавления конвекция усиливается. Удваивается по сравнению с сухой мантией и интенсивность всех связанных с ней процессов, включая плавление. (В случае сухой мантии максимальное обеднение базальтовым компонентом на порядок ниже, т.е. ее плотностной эффект пренебрежимо мал по сравнению с тепловым расширением, соответственно интенсивность конвекции вдвое меньше). Заметим, что влияние обеднения базальтовым компонентом на интенсивность конвекции отмечалось и другими исследователями, например [Sparks, Parmantier, 1993; Schmeling, 2000].

В целом, картина петрологической зональности верхней мантии, формирующейся в восходящем потоке в результате плавления, миграции и замерзания расплава, выглядит разумно и не противоречит имеющимся геолого-геофизическим данным (напомним, что на поверхности восходящий поток верхнемантийной конвекции формирует эпиконтинентальный осадочный бассейн [Lunev, Lapkovsky, 2018]).

Некоторые из полученных результатов, по-видимому, требуют уточнения. Прежде всего, сомнение вызывает довольно глубокая локализация зоны замерзания расплава. Дело в том, что при малой концентрации расплава перенос им тепла оказывается так же мал, несмотря на высокую скорость его движения, мало и его влияние на среднюю температуру ячейки с использованным шагом сетки $3.0 \times 1.5$ км (т.е. объемом порядка 10 км³ $^{3}$. То же относится и к выделению тепла при 
замерзании расплава. А именно это общее для ячейки значение температуры используется при определении фазового состояния вещества, включая расплав. В действительности температура быстро движущегося расплава, конечно, гораздо выше такого среднего значения, так что замерзать он должен на более высоких уровнях. В данном случае (для принятого уравнения состояния) естественно ожидать, что базальтовый расплав будет накапливаться и замерзать (по крайней мере частично) у подошвы континентальной коры или внутри нее - там, где обнуляется его плавучесть и прекращается подъем. Для того чтобы описать этот эффект, необходимо либо существенно сгустить расчетную сетку (используя, например, для области присутствия расплава расчет динамики расплава на вложенной сетке), либо каким-то специальным образом учесть специфику переноса тепла расплавом. Кроме того, как уже говорилось, желательно существенно (на порядок) увеличить детальность расчетной сетки, чтобы получить структуру миграции расплава в виде каналов.

Также желательно в дальнейшем ввести в модель определение состава образующегося расплава и его изменения и более аккуратно учитывать изменение состава матрицы в результате его сепарации и замерзания.

\section{5. ЗАКЛЮЧЕНИЕ}

Приведенные результаты моделирования ни в коем случае не претендуют на окончательность, они, несомненно, нуждаются в уточнении и конкретизации. Однако они в общем соответствуют известным геолого-геофизическим данным о строении литосферы под развивающимися эпиконтинентальными осадочными бассейнами и в рамках своей детальности согласуются с результатами, полученными путем расчета реакций между компонентами мантийного вещества. Это говорит об адекватности предложенной модели декомпрессионного плавления и поведения расплава, при том что она остается феноменологической и соответствует первому приближению, органично дополняя развиваемую нами модель мантийной конвекции. Важно также то, что предложенная модель как раз допускает возможность уточнения, причем, помимо детальности, именно в той части, где возможно уточнение на основании реальных контролируемых экспериментов с веществом, устанавливающих параметры фазовых и химических превращений, их плотностных и тепловых эффектов, а также - динамики жидкой фазы, в зависимости от ее состава и состава окружающей среды.

\section{6. ЛИТЕРАТУРА / REFERENCES}

Ablesimov N.E., Zemtsov A.N., 2010. Relaxation Effects in Non-Equilibrium Condense Systems. In: Basalts: from Eruption up to Fiber. Institute of Tectonics and Geophysics FB RAS, Moscow, 400 p. (in Russian) [Аблесимов Н.E., Земцов A.Н. Релаксационные эффекты в неравновесных конденсированных системах // Базальты: от извержения до волокна. М.: ИТиГ ДВО РАН, 2010. 400 с.].
Ablesimov N.E., Lipatov V.G., Taltykin Yu.V., Berdnikov N.V., 1988. The Role of Volatiles in Generation of Deep Geophysical Anomalies. Journal of Physics of the Earth 36 (2), S191S196. https://doi.org/10.4294/jpe1952.36.Proceeding 2_S191.

Batanova V.G., Savelieva G.N., 2009. Melt Migration in the Mantle beneath Spreading Zones and Formation of Replacive Dunites: A Review. Russian Geology and Geophysics 50 (9), 763-778. https://doi.org/10.1016/j.rgg.2009.08.008.

Blokhin A.M., Dorovsky V.N., 1994. Problems of Mathematical Modeling in the Theory of Multi-Velocity Continuum. United Institute of Geology, Geophysics and Mineralogy SB RAS, Institute of Mathematics, Novosibirsk, 183 p. (in Russian) [Блохин A.M., Доровский В.Н. Проблемы математического моделирования в теории многоскоростного континуума. Новосибирск: ОИГГМ СО РАН, Институт математики, 1994. 183 с.].

Gorbachev N.S., Kostyuk A.V., Sultanov D.M., 2017. Phase Relations of Fluid-Containing Peridotite at Undercritical and Supercritical Pressures (Experimental Data). In: PhysicalChemical and Petrophysical Researches in the Earth's Sciences. Proceedings of the Eighteenth International Conference (Moscow, October 2-4, Borok, October 6, 2017). Moscow: IGEM RAS, p. 66-69 (in Russian) [Горбачев H.C., Костюк А.В., Султанов Д.М. Фазовые соотношения флюидсодержащего перидотита при докритических и сверхкритических давлениях (по экспериментальным данным) // Физико-химические и петрофизические исследования в науках о Земле: Материалы Восемнадцатой международной конференции (Москва, 2-4, Борок, 6 октября 2017 г.). М.: ИГЕМ РАН, 2017. С. 66-69].

Kelemen P., Hirth G., Shimizu N., Spiegelman M., Dick H., 1997. A Review of Melt Migration Processes in the Adiabatically Upwelling Mantle beneath Oceanic Spreading Ridges. Philosophical Transactions of the Royal Society A: Mathematical, Physical and Engineering Sciences 355, 283-318. https://doi.org/10.1098/rsta.1997.0010.

Keller T., Katz R.F., 2016. The Role of Volatiles in Reactive Melt Transport in the Asthenosphere. Journal of Petrology 57 (6), 1073-1108. https://doi.org/10.1093/petrology/ egw030.

Keller T., Katz R.F., Hirschmann M.M., 2017. Volatiles beneath Mid-Ocean Ridges: Deep Melting, Channelised Transport, Focusing, and Metasomatism. Earth and Planetary Science Letters 464, 55-68. https: //doi.org/10.1016/j.epsl. 2017.02.006.

Keller T., Suckale J., 2019. A Continuum Model of MultiPhase Reactive Transport in Igneous Systems. Geophysical Journal International 219 (1), 185-222. https://doi.org/10. 1093 /gji/ggz287.

Lichtenberg T., Keller T., Katz R.F., Golabek G.J., Gerya T.V., 2019. Magma Ascent in Planetesimals: Control by Grain Size. Earth and Planetary Science Letters 507, 154-165. https:// doi.org/10.1016/j.epsl.2018.11.034.

Litasov K.D., 2011. Physicochemical Conditions for Melting in the Earth's Mantle Containing a C-O-H Fluid (from Experimental Data). Russian Geology and Geophysics 52 (5), 475-492. https://doi.org/10.1016/j.rgg.2011.04.001. 
Lunev B.V., Lapkovsky V.V., 2018. The First-Approximation Model Showing the Occurrence of Epicontinental Sedimentary Basins Due to Convective Instability of the Thermal Lithosphere. Geodynamics \& Tectonophysics 9 (4), 13631380 (in Russian) [Лунёв Б.В., Лапковский В.В. Модель первого приближения формирования эпиконтинентальных осадочных бассейнов вследствие конвективной неустойчивости термической литосферы // Геодинамика и тектонофизика. 2018. Т. 9. № 4. С. 1363-1380]. https://doi.org/10.5800/GT-2018-9-4-0400.

McKenzie D., 1984. The Generation and Compaction of Partially Molten Rock. Journal of Petrology 25 (3). 713-765. https://doi.org/10.1093/petrology/25.3.713.

Perepechko Yu.V., Sharapov V.N., 2014. Conditions of Appearance of the Asthenospheric Layer under Upper Mantle Convection. Doklady Earth Sciences 457, 901-904. https:// doi.org/10.1134/S1028334X14070241.

Polyansky O.P., Babichev A.V., Reverdatto V.V., Korobeynikov S.N., 2012. Formation and Upwelling of Mantle Diapirs through the Cratonic Lithosphere: Numerical Thermomechanical Modeling. Petrology 20, 120-137. https://doi. org/10.1134/S086959111202004X.

Polyansky O.P., Korobeinikov S.N., Babichev A.V., Reverdatto V.V., Sverdlova V.G., 2014. Numerical Modeling of Mantle Diapirism as a Cause of Intracontinental Rifting. Izvestiya, Physics of the Solid Earth 50, 839-852. https://doi.org/10. 1134/S1069351314060056.

Polyansky O.P., Prokopiev A.V., Koroleva O.V., Tomshin M.D., Reverdatto V.V., Babichev A.V., Sverdlova V.G., Vasiliev D.A., 2018. The Nature of the Heat Source of Mafic Magmatism during the Formation of the Vilyui Rift Based on the Ages of Dike Swarms and Results of Numerical Modeling. Russian Geology and Geophysics 59 (10), 1217-1236. https://doi. org/10.1016/j.rgg.2018.09.003.

Polyansky O.P., Prokopiev A.V., Koroleva O.V., Tomshin M.D., Reverdatto V.V., Selyatitsky A.Yu., Travin A.V., Vasiliev D.A., 2017. Temporal Correlation between Dyke Swarms and Crustal Extension in the Middle Palaeozoic Vilyui Rift Basin, Siberian Platform. Lithos 282-283, 45-64. https://doi.org/ 10.1016/j.lithos.2017.02.020.

Polyansky O.P., Reverdatto V.V., Babichev A.V., Sverdlova V.G., 2016. The Mechanism of Magma Ascent through the Solid Lithosphere and Relation between Mantle and Crustal Diapirism: Numerical Modeling and Natural Examples. Russian Geology and Geophysics 57 (6), 843-857. https://doi. org/10.1016/j.rgg.2016.05.002.
Rudge J.F., Bercovici D., Spiegelman M., 2011. Disequilibrium Melting of a Two Phase Multicomponent Mantle. Geophysical Journal International 184 (2), 699-718. https:// doi.org/10.1111/j.1365-246X.2010.04870.x.

Saxena S.K., Eriksson A.G., 1985. Anhydrous Phase Equilibria in Earth's Upper Mantle. Journal of Petrology 26 (2), 378-390. https://doi.org/10.1093/petrology/26.2.378.

Schmeling H., 2000. Partial Melting and Melt Generation in a Convecting Mantle. In: N. Bagdassarov, D. Laporte, A.B. Thompson (Eds). Petrology and Structural Geology. Vol. 11. Springer, Dordrecht, p. 114-178. https://doi.org/ 10.1007/978-94-011-4016-4_5.

Schmeling H., 2006. A Model of Episodic Melt Extraction for Plumes. Journal of Geophysical Research: Solid Earth 111 (B3). https://doi.org/10.1029/2004JB003423.

Schmeling H., 2010. Dynamic Models of Continental Rifting with Melt Generation. Tectonophysics 480 (1-4), 33-47. https://doi.org/10.1016/j.tecto.2009.09.005.

Sharapov V.N., Perepechko Yu.V., Mazurov M.P., 2006. Mantle-Crust Magmatic-Fluid Systems in Spreading Zones. Russian Geology and Geophysics 47 (12), 1326-1344.

Sobolev S.V., Babeyko A.Yu., 1994. Modeling of Mineralogical Composition, Density and Elastic Wave Velocities in Anhydrous Magmatic Rocks. Surveys in Geophysics 15, 515-544. https://doi.org/10.1007/BF00690173.

Sobolev A.V., Krivolutskaya N.A., Kuzmin D.V., 2009. Petrology of the Parental Melts and Mantle Sources of Siberian Trap Magmatism. Petrology 17, 253. https://doi.org/10.11 34/s0869591109030047.

Sparks D.W., Parmantier E.M., 1993. The Structure of Three-Dimentional Convection beneath Oceanic Spreading Centres. Geophysical Journal International 112 (1), 81-91. https://doi.org/10.1111/j.1365-246X.1993.tb01438.x.

Sparks D.W., Parmantier E.M., 1994. Chapter 4 The Generation and Migration of Partial Melt beneath Oceanic Spreading Centers. International Geophysics 57, 55-76. https:// doi.org/10.1016/S0074-6142(09)60092-9.

Turner A.J., Katz R.F., Behn M.D., Keller T., 2017. Magmatic Focusing to Mid-Ocean Ridges: The Role of Grain-Size Variability and Non-Newtonian Viscosity. Geochemistry Geophysics Geosystems 18 (12), 4342-4355. https://doi.org/ 10.1002/2017GC007048.

Zhuse T.P., 1981. The Role of Compressed Gases as Dissolvents. Nedra, Moscow, 161 p. (in Russian) [Жузе Т.П. Роль сжатых газов как растворителей. М.: Недра, 1981. 161 c.]. 